\title{
A PERCEPÇÃO NA AÇÃO INTENCIONAL
}

PERCEPTION IN INTENTIONAL ACTION

Daniel De Luca Noronha*

\section{RESUMO}

O objetivo desse artigo é explorar o papel da percepção no domínio do comportamento intencional. No primeiro tópico, o artigo leva em conta a teoria tradicional da ação, tal como proposta por Donald Davidson. De acordo com essa teoria, a percepção causa as atitudes proposicionais; essas atitudes, por sua vez, fornecem as razões que justificam as ações. Nesse sentido, a percepção é concebida como crença perceptiva e, assim, seus conteúdos são constituídos por conceitos. Entretanto, no segundo tópico, eu argumento que essa linha de pensamento não faz justiça à percepção na ação. Isso porque a percepção não precisa estar conectada às atitudes proposicionais para figurar nas ações intencionais. No terceiro tópico, eu proponho que percepção e ação são profundamente interdependentes. Por fim, eu argumento que as relações recíprocas entre percepção e ação são fundamentais para a filosofia da ação.

PALAVRAS-CHAVE: Percepção. Ação. Atitudes proposicionais.

\section{ABSTRACT}

The aim of this paper is to explore the role of perception in the domain of intentional behavior. On the first topic, the paper takes into account the traditional theory of action, as proposed by Donald Davidson. According to this theory, perception causes propositional attitudes; these attitudes, in turn, provide the reasons that justify actions. In this sense, perception is conceived as perception-belief, and so its contents are constituted by concepts. However, on the second topic I argue that this line of thought does not make justice to perception in action. This is because perception needs not be connected to propositional attitudes in order to figure on the intentional actions. On the third topic, I propose that perception and action are deeply interdependent. I finish by arguing that the reciprocal relations between perception and action are critical to philosophy of action.

KEYWORDS: Perception. Action. Propositional attitudes.

\footnotetext{
* Doutor em Filosofia pela UFMG. Instituição: Faculdade Jesuíta de Filosofia e Teologia - FAJE. Email: deluca.11@gmail.com
} 


\section{A teoria tradicional da ação}

Qual é o papel da percepção no comportamento intencional? De início, vejamos como um evento físico, tal como um movimento corporal, pode ser concebido como uma ação intencional. De acordo com Donald Davidson, para que um movimento corporal seja concebido como uma ação, ele deve poder ser descrito por meio de atitudes proposicionais que, do ponto de vista do agente, explicam sua ação. As atitudes proposicionais relevantes para a explicação da ação são o desejo e a crença. Enquanto o conteúdo do desejo estabelece os objetivos da ação, o conteúdo da crença fornece os meios para a realização desses objetivos. É importante notar que Davidson entende a atribuição de atitudes proposicionais como uma condição necessária da compreensão da ação intencional. Ou seja, um movimento ao qual não é possível atribuir atitudes proposicionais não é, propriamente, uma ação humana (DAVIDSON, 1974, p. 229).

As atitudes proposicionais organizam-se em uma rede de relações inferenciais. Davidson evidencia essas relações retomando o conceito tradicional de raciocínio prático: as crenças e os desejos são representados como premissas cuja conclusão é a própria ação (DAVIDSON, 1963, p. 9). O raciocínio prático apresenta uma garantia de que desejos, crenças e ações sejam coerentes entre si. Em relação a isso, para que os desejos e as crenças possam satisfazer aos critérios normativos a que estão submetidos, o conteúdo dessas atitudes deve ser de natureza conceitual. Além das relações racionais entre as atitudes proposicionais, Davidson entende que elas devem exercer um papel causal. Isso porque, sem recorrer à causação, não há como distinguir os casos em que o agente tem razões para agir, mas não age de acordo com essas razões, dos casos em que o agente efetivamente age com base nessas razões. Em suma, é justamente porque a razão está relacionada de forma causal com a ação que a ação pode ser explicada por referência à razão (DAVIDSON, 1963, p. 9).

Note-se, no entanto, que as atitudes proposicionais não são a única fonte causal das ações. Evidentemente, elas situam-se no início de um processo que se desenrola mediante um movimento corporal tendo em vista um objetivo a ser alcançado. O movimento corporal é também causalmente constrangido pelas circunstâncias de um entorno físico particular, com as quais os agentes têm de lidar para realizarem seus objetivos. Assim, a formação de atitudes proposicionais ocorre no raciocínio e no planejamento das ações. Nesse nível, os agentes não costumam estimar, de maneira prévia ao movimento, os diversos ajustes corporais que devem fazer diante das circunstâncias do entorno físico. Por certo, os agentes errariam com muito mais 
freqüência do que efetivamente erram nos seus movimentos se tivessem que calcular todos esses ajustes (MILNER; GOODALE, 2006, p. 177). Já no decorrer da ação, o corpo responde imediatamente aos estímulos do entorno físico, realizando alterações neurológicas, mudanças no tecido muscular entre outros micro-ajustes corporais. Entretanto, note-se que nada disso parece decorrer de uma intenção explícita do agente. $O$ agente simplesmente não tem consciência de todas essas alterações, elas não decorrem de um cálculo racional (MILNER; GOODALE, 2006, p. 175-176). Em suma, o processo racional de escolha e planejamento da ação não é suficiente para controlar e monitorar os movimentos do corpo em ação.

Dessas considerações surge uma imagem bifurcada da ação. De um lado, o nível superior que chamamos nível pessoal. Esse é o nível das atitudes proposicionais, cujo conteúdo é mobilizado pelo agente em um campo inferencial e que serve de fonte explicativa da ação. De outro lado, o nível inferior, chamado subpessoal. Nesse nível são produzidos ajustes automáticos, a que o agente não tem acesso nem controle consciente. Portanto, esse nível é irrelevante para a explicação da ação. Teorias tradicionais da ação, como a teoria de Davidson, assume essa bifurcação e restringe sua análise ao nível superior. Davidson expressa da seguinte maneira essa bifurcação:

\footnotetext{
We are stuck with our two main ways of describing and explaining things, one which treats objects and events as mindless, and the other which treats objects and events as having propositional attitudes. I see no way of bridging the gap by introducing an intermediate vocabulary for talking about non-human animals. (DAVIDSON, 2003, p. 697).
}

Entretanto, como procurarei mostrar, esse quadro não é compulsório. Além disso, essa bifurcação deixa na sombra um explanandum fundamental da teoria da ação. Estarei menos interessado em cruzar a fronteira das espécies do que tentar mostrar a importância desse vocabulário intermediário para lidar com uma dimensão ligada à própria ação humana.

\section{A percepção como explanandum}

A estrutura bifurcada na teoria tradicional da ação, que aqui vimos nos seus traços principais, não acomoda um fenômeno central do comportamento intencional. Vejamos agora, com algum vagar, qual é esse fenômeno. Uma primeira aproximação consiste em perguntar pelo lugar da percepção dentro do quadro dessa teoria. Quanto a isso, Davidson afirma o seguinte: 
The physical world assails our senses, and the outcome is a sophisticated conceptual picture of that world; by appealing to that picture we reason how to achieve our purposes through action. In perception the physical world causes thought; in action thought brings changes in our physical environment. (DAVIDSON, 2001, p. 277).

Entre a percepção, que captura o estímulo distal, e a resposta comportamental, Davidson estabelece o pensamento reflexivo como um medium dessa relação. Nessa perspectiva, para que a percepção tenha algum papel na explicação da ação intencional ela deve ser tomada como crença perceptiva, como algo que envolve conceitos. Ou seja, sem a conexão com a crença, a percepção não tem lugar na explicação da ação. Dentro desse quadro bifurcado, a percepção segue, assim, a reboque das atitudes proposicionais. Para os nossos propósitos, é importante avaliar esse movimento davidsoniano de assimilar a percepção às atitudes proposicionais como a crença no domínio da ação.

A objeção importante ao argumento de Davidson é que o potencial explicativo das atitudes proposicionais não é tão abrangente para envolver tudo que importa para a explicação da ação intencional. Considere-se o problema do movimento impróprio colocado por Perry et al. (1993): o agente pode ter crenças verdadeiras e desejos passíveis de ser realizados; apesar disso, pode executar o movimento de forma inapropriada - de tal maneira que não realiza o objetivo pretendido. Certamente, pode-se explicar o erro do movimento corporal mediante uma descrição subpessoal. Nesse caso, tal como afirma a teoria tradicional da ação, temos uma descrição irrelevante para a explicação da ação intencional.

Não obstante, considere-se que o movimento inapropriado decorreu de uma falha perceptual, da qual o agente se torna consciente no momento mesmo em que executa equivocadamente o movimento. Temos algo que, do ponto de vista do agente, parece figurar como um elemento central da ação. Entretanto, as atitudes proposicionais em questão não parecem ser suficientes para explicar esse fenômeno. A explicação pertinente para essa situação requer tematizar a percepção no decorrer da ação. Noutros termos, o intérprete deve levar em conta o ponto de vista do agente. Com efeito, se o desenrolar das ações varia com a perspectiva perceptual do agente, não é então suficiente, para fins de explicação da ação, ter presente somente o objeto da sua percepção. São, antes, os modos de perceber um objeto, determinados pela posição cambiante do agente em uma dada situação, que devem figurar nessa explicação.

Esse mesmo ponto pode ser focalizado na predição de um comportamento intencional. Com base na atribuição de desejos e crenças ao agente, espera-se um determinado comportamento. No entanto, considere-se que no momento da ação o agente dirige seus movimentos corporais a objetos diferentes do que se poderia esperar a partir da atribuição 
realizada. Ou então, que o agente realiza movimentos estranhos, que não parecem se direcionar a quaisquer objetos ou eventos do mundo. Em ambos os casos, há uma quebra de expectativa daquilo que seria perceptualmente relevante para o movimento do agente. Com base nisso, pode-se rever a atribuição inicial de atitudes proposicionais. Nesse sentido, aquilo que no mundo captura a atenção perceptual do agente no curso do seu movimento corporal é um elemento chave da explicação da ação. Entretanto, a posse de atitudes proposicionais não parece ser uma condição necessária para que um ser perceba algo em função do que ele age. Tampouco essa posse é uma condição necessária para que um intérprete seja sensível aos objetos da atenção perceptual do outro, vale dizer, a capacidade de rastrear tais objetos é também uma capacidade perceptual e não depende da posse de atitudes proposicionais. Portanto, em que pese o fato de esse fenômeno ser parte da ação intencional, não é certo que dependemos da atribuição de atitudes proposicionais para explica-lo.

Esses problemas apontam para a necessidade de se pensar uma conexão direta (nãomediada) entre percepção e movimento corporal como um elemento da explicação da ação. Ou seja, diferentemente do que Davidson afirma, parece que a percepção não precisa estar ligada à crença para ter lugar no âmbito da ação intencional. Isso porque parte do que percebemos no curso de nossas ações exerce algum tipo de controle sobre nossos movimentos, independentemente das atitudes proposicionais.

A essa altura podemos esclarecer nosso explanadum. Trata-se da relação entre percepção e movimento corporal direcionado a algo do mundo. Talvez essa relação seja intuitiva. Parece natural afirmar que parte das informações perceptuais é utilizada nas nossas ações, ou seja, para a realização dos nossos objetivos. Com efeito, o sucesso das nossas ações pode ser parcialmente explicado pela validade dessas informações perceptuais, nas quais aprendemos a confiar desde muito cedo na nossa ontogênese. Entretanto, o problema é que esse explanandum não tem lugar no quadro bifurcado que foi colocado. Tudo indica que, por um lado, não é possível compreender essa percepção como se fosse um passo no interior do domínio das atitudes proposicionais. Por outro lado, essa percepção não se coloca no nível subpessoal, controlando e monitorando micro-ajustes corporais que, comumente, não se colocam no foco da atenção dos agentes. Como temos visto, os casos em tela são aqueles sobre os quais o agente é consciente na sua percepção, de uma maneira relevante para o seu próprio comportamento. 


\section{Nível Intermediário: as conexões dinâmicas entre percepção e ação}

Um caminho para lidar com esse explanandum é cobrir o gap deixado pelo quadro bifurcado. Entre o nível superior das atitudes proposicionais e o nível inferior de ajustes subpessoais, há um nível intermediário que pretendo explorar. Nesse nível intermediário encontra-se algo como um conjunto de capacidades práticas que nos permite estabelecer, por assim dizer, rotas no mundo para a realização dos nossos objetivos. Essas capacidades envolvem um determinado tipo de compreensão das estruturas espaciais, que não pode ser separado do nosso envolvimento pessoal e ativo com o mundo. Ou seja, uma compreensão que resulta do nosso enraizamento, de nossa habituação com objetos e eventos no ambiente. Algo que começa a ser gerado desde muito cedo na nossa ontogênese, na medida em que nos engajamos em atividades exploratórias sobre objetos. Justamente quando começamos a aprender sobre os limites e as possibilidades de nossos movimentos pelo mundo.

A literatura mostra algumas abordagens desse campo que estamos evidenciando. Podemos citar as análises de Christopher Peacocke (1993) e John Campbell (1993) sobre a ideia de física primitiva, i.e., a maneira pela qual a compreensão do mundo está diretamente relacionada com nossas ações. A física primitiva envolve um conjunto de expectativas acerca do comportamento dos fenômenos físicos que nos permite exercer um controle sobre eles e, deste modo, navegar com algum sucesso pelo mundo. Por exemplo, ao jogar um objeto para cima, um agente antecipa a sua queda e age em função dessa expectativa. Para que o agente seja capaz de criar essa expectativa, não é necessário um conhecimento explícito acerca da lei da gravidade. Basta que ele seja capaz de detectar determinadas uniformidades nas diferentes situações em que se encontra.

Outro exemplo interessante dessa compreensão prática encontra-se num trabalho de Adrian Cussins (2003, p. 142). Ele apresenta dois modos disponíveis a um motociclista de saber a velocidade de sua moto. No primeiro caso, o motociclista sabe a velocidade a partir do que ele observa no velocímetro, dentro de um determinado padrão de medida. Ele é capaz de saber que nessa mesma velocidade poderia estar um carro ou um jipe em outra estrada, ou seja, é capaz de generalizar esse conhecimento. Não obstante, parece haver outro tipo de saber, nesse caso prático e não-proposicional, que é totalmente limitado às circunstâncias físicas em que o agente se encontra. Trata-se de um tipo de saber que depende do modo específico como ele interage com o mundo. Ora, mesmo sem ter acesso ao velocímetro, o motociclista sente o trepidar da roda, o vento que sopra contra o seu corpo, percebe mudanças rápidas que ocorrem 
na periferia do seu campo visual e assim por diante. A partir da sensibilidade a esse conjunto de informações, o motociclista realiza diversos tipos de ajustes enquanto percorre o trajeto. Em relação a esse saber prático e não-proposicional, Adrian Cussins afirma o seguinte: “As you move you are not, or need not, be guided by truth, but you are, nevertheless, guided; you are guided by the mundane structure of the activity-space around you." (CUSSINS, 2003, p. 144).

Assim temos alguns elementos para afirmar que o agente não precisa de conceitos para ter uma experiência com esse tipo de conteúdo. Ou seja, ele pode agir a partir desse tipo de conteúdo perceptual, sem que para isso tenha a necessidade de possuir capacidades conceitualmente estruturadas. Em resumo, trata-se de um conteúdo não conceitual. Não obstante, compreenderíamos mal essa capacidade se a pensarmos simplesmente como a capacidade de fazer ajustes automáticos e cegos de comportamento. Como afirma John Campbell, essa capacidade requer o domínio das relações entre percepção e ação (CAMPBELL, 1993, p. 88). Tal capacidade inclui reorientação flexível em função de alterações constantes no ambiente, sobretudo diante de situações imprevistas. Também inclui uma rede de conexões entre agente e ambiente da qual surgem expectativas que orientam as ações desses agentes para a realização dos seus objetivos. Essa capacidade não se faz presente, portanto, no comportamento de criaturas muito simples, como bactérias que se orientam pelo polo norte, para as quais determinadas alterações no ambiente são suficientes para impedir a realização da ação.

Até aqui temos uma ideia de uma capacidade prática que requer a conexão entre percepção e ação. Entretanto, essa conexão deve ser investigada no pormenor. De forma mais precisa, o que significa agir com base no que se percebe? Um conceito que elucida parte dessa conexão é o de affordances, postulado por Jerome Gibson em sua abordagem ecológica da percepção visual (GIBSON, 1986, p. 127-143). Affordances são recursos que o ambiente oferece a uma criatura e que são pertinentes para o seu ciclo de vida. Basicamente, para Gibson, o ambiente não é, no mais das vezes, percebido de forma neutra. A percepção do ambiente submete-se em larga medida aos objetivos que o organismo pretende atingir. Nas ações dirigidas a objetos e eventos do mundo, as propriedades dos objetos são capturadas em função de sua potencialidade motora. Essas propriedades são apresentadas enquanto capacidades que nos permitem agir no ambiente. Assim, perceber é aprender, em sentido prático, de que forma o ambiente estrutura as possibilidades de movimento do agente. Podemos dizer que nessa relação íntima com a ação, a percepção nos coloca em contato menos com uma realidade objetiva e independente, que poderíamos alcançar no nível das atitudes proposicionais, do que com o 
mundo enquanto espaço em que realizamos nossas ações. $\mathrm{O}$ ambiente figura enquanto uma instância mediadora das nossas ações, que permite certos movimentos e que impede outros.

Parte do que percebemos é, portanto, utilizado nas nossas ações. Mas é fundamental notar que o inverso também ocorre. Isso porque não seria possível para o agente selecionar o que é relevante para a sua ação sem que ele mesmo realizasse movimentos corporais pelos quais expusesse seus órgãos sensoriais sob a influência das affordances. A ideia chave é que a percepção, ela mesma, consiste em uma forma de ação, ou seja, não é possível perceber sem um corpo que se movimenta. Trata-se de uma atividade que se estende no tempo, que requer engajamento e exploração dos objetos. A percepção não é, portanto, como uma espécie de fotografia, da qual o sujeito percipiente é um mero expectador, em que todos os detalhes de uma cena são capturados ao mesmo tempo. Algumas pesquisas mostram que somos perceptualmente insensíveis a determinadas alterações no campo visual (NÖE, 2004, p. 51-52). Quando percebemos, focamos nossa atenção em determinados aspectos de uma cena, enquanto deixamos os outros na sombra do campo visual. A ideia da percepção como forma de ação conduz ao que Alva Nöe chama de domínio de contingências sensório motoras. Um domínio sobre o modo pelo qual as alterações no campo visual co-variam com que o sujeito faz - para dar um exemplo simples, se o agente vira sua cabeça para a esquerda, ele não estranha que o objeto que se encontrava à sua frente agora se encontra à direita do seu campo visual.

Essas considerações nos mostram que há uma interdependência na relação entre percepção e movimento corporal. O papel que Davidson reserva para a percepção no campo da ação é apenas uma parte, de resto sofisticada por envolver conceitos, dessa interação complexa. Com efeito, uma vez que os movimentos do indivíduo são sensíveis aos impactos que o ambiente causa no seu aparato perceptivo, pode-se dizer que o comportamento é uma resposta ao estímulo do mundo. Ao mesmo tempo, dado que os estímulos que o indivíduo acessa só são possíveis mediante movimentos precedentes, podemos dizer que o comportamento ele mesmo participa do processo de causação ligado ao estímulo.

Tais conexões entre percepção e ação foram bem esclarecidas por Susan Hurley (1998). Hurley argumenta que devemos caracteriza-las como ciclos de estímulo-resposta, nos quais perceber e agir interagem entre si de maneira dinâmica. Para ela, são justamente esses ciclos, não apenas partes deles, que explicam o processo de ajuste de nível superior ou pessoal do indivíduo no mundo. Ao tentar entender o lugar da mente no mundo, é comum nos atermos somente à direção do estímulo para a resposta. Daí, segundo Hurley, 
(...) we tend to ignore the function from output back to input, and the way environments transform and reflect outputs from the human organism. The two functions are not only of comparable complexity, but are causally continuous. To understand the mind's place in the world, we should study these complex dynamic processes as a system, not just the truncated internal portion of them. (HURLEY, 1998, p. 2)

As repercussões desse argumento para a filosofia da ação parecem evidentes e de amplo alcance. Com efeito, torna-se difícil traçar uma fronteira nítida demarcando aonde a mente termina e o mundo começa. Não se trata somente da ideia de uma mente corporificada, mas também de uma mente engajada ou enraizada no ambiente, com o qual está em constante conexão ativa. No que importa para a filosofia da ação, temos algo como um acoplamento sensório-motor que se dá no nível pessoal. Ou seja, o ambiente, que é constantemente alterado e moldado pelos agentes, também é parte daquilo que controla diretamente seus movimentos corporais. Para evidenciar esse ponto, basta pensar nos instrumentos do mundo que se colocam diretamente a serviço das ações. Evidentemente, tais instrumentos são em princípio descontínuos com o corpo. Mas eles são comumente apropriados e acoplados ao processo cognitivo pelo qual o agente interage com o ambiente. Nesse caso a mente corporificada, por assim dizer, se estende por meio desses instrumentos.

Do que foi colocado emerge a ideia de um agente dotado de habilidade prática para rastrear as conexões entre o que faz e o que percebe. Com base nisso ele assume diversas perspectivas com relação aos objetos para realizar seus objetivos. Assumir uma perspectiva significa compreender de maneira prática as conexões entre percepção e ação e, por meio dessa compreensão, estabelecer diferentes modos de acessar o objeto tendo em vista os objetivos que se têm em vista. Daí podemos dizer que o agente tem acesso a um conteúdo perceptual. Mas, como vimos, dado que o agente não precisa da posse de conceitos para ter uma experiência com esse tipo de conteúdo, trata-se de um conteúdo não conceitual.

Enfim, esse modo de acessar o mundo, que requer o domínio das relações dinâmicas entre percepção e movimento corporal, deve ser entendido como parte essencial do comportamento intencional. Dessa maneira, estamos em condições de contornar um dos aspectos centrais da bifurcação apontada por Davidson. Do que vimos resulta que possuir conceitos ou atitudes proposicionais não parece ser uma condição necessária para se possuir intencionalidade. Uma criatura intencional é aquela capaz de, basicamente, traçar rotas em direção aos objetos. É certo que um modo superior de traçar essas rotas se dá por meio de atitudes proposicionais. Mas, vale insistir, esse não é o único modo. O outro modo ocorre pela 
assunção pessoal de perspectivas pelas quais os objetos se nos apresentam. Sem demandar a posse de conceitos, esse tipo de acesso ao mundo é direcionado aos objetivos que o agente pretende atingir. Parece razoável supor que essa capacidade é algo que seres humanos compartilham com outros primatas como chimpanzés. É igualmente certo, no entanto, que organismos simples como plantas e bactérias não possuem essa capacidade.

\section{Conclusão: repercussões para a filosofia da ação}

De que modo essas relações entre percepção e ação alteram a concepção de ação intencional proposta pela teoria tradicional da ação? Vamos retomar a concepção de ação intencional proposta por Davidson: para que um movimento corporal seja concebido como uma ação, ele deve poder ser descrito por meio de atitudes proposicionais que, do ponto de vista do agente, explicam sua ação. Aqui temos uma base para pensarmos o papel do agente acerca de sua ação. O ponto que distingue a ação intencional de meros movimentos reflexos é, então, a capacidade de o agente oferecer razões que justifiquem a sua ação. Ou seja, dentro dessa concepção de comportamento intencional, atribui-se ao agente a capacidade de dar um passo atrás no curso de sua ação e refletir acerca das suas atitudes proposicionais que poderiam justifica-la. Aquém dessa capacidade reflexiva, temos meros movimentos corporais desprovidos de intencionalidade.

Ora, tendo em vista o nível intermediário já visto, a concepção de ação intencional que emerge é mais básica ou primitiva. De acordo com essa concepção, para que um movimento corporal seja concebido como ação intencional é suficiente que o agente seja capaz de assumir diferentes perspectivas acerca de uma situação e, desse modo, ajustar-se de maneira flexível para alcançar realizar os seus objetivos. O agente possui um domínio prático acerca de seu corpo e, de modo básico, é capaz de distinguir as ocasiões em que é passivo diante de alterações corporais, das ocasiões em que se sente no controle ou, em suma, que se percebe enquanto agente. Estando no controle das conexões entre percepção e ação, ele é capaz de diferenciar, em seu próprio corpo, movimentos não intencionais de movimentos intencionais, bem como antecipar os efeitos desses últimos.

Enfim, ao enfraquecer as condições pelas quais um movimento pode ser considerado como intencional, abrimos o leque para atribuir intencionalidade a agentes sem capacidades reflexivas, como crianças em determinada fase da ontogênese, ou ainda alguns primatas superiores. O principal impacto de se ampliar as condições pelas quais uma ação pode ser 
considerada intencional é superar o aspecto restritivo da concepção tradicional e, assim, contornar o problema da perda de um fenômeno constitutivo da ação intencional.

Por fim, vamos fazer um resumo do que foi colocado. Começamos por problematizar a estrutura bifurcada da ação intencional proposta pela filosofia tradicional da ação. Tal como foi colocado, o problema é que ao associar a ação intencional às atitudes proposicionais, ou ainda à capacidade de o agente refletir sobre elas, deixa-se de lado o transcorrer da ação ou o movimento corporal. Ao investigarmos esse domínio, deparamo-nos com a habilidade prática de os agentes relacionarem percepção e ação como parte central do comportamento intencional. Diferentemente da linha divisória adotada por Davidson, portanto, surge uma capacidade perceptual que se coloca entre ajustes automáticos de comportamento e capacidades conceituais.

O interesse em pesquisar essa capacidade não está ligado somente ao fato de que se trata, ao que tudo indica, de uma etapa essencial da nossa ontogênese, pela qual precisamos passar para chegarmos ao nível de planejar racionalmente nossas ações. Além disso, é algo que figura na estrutura mesma da ação, vale dizer, algo do qual o ser humano é consciente na sua percepção e que participa das condições de sucesso ou fracasso da sua ação. Trata-se, enfim, de um legítimo explanandum da filosofia da ação.

\section{REFERÊNCIAS}

ANSCOMBE, Elizabeth. Intention. Harvard University Press, 1975.

BACH, Kent. A representacional theory of action. Philosophical studies 36, 1978.

BAILLARGEON, Renée; LUO, Yuyan. When the ordinary seems unexpected: evidence for incremental physical knowledge in young infants. Cognition, 95, p. 297-328, 2005.

BERMUDEZ, Jose Luiz. Ecological perception and the notion of a nonconceptual point of view. In. The body and self. Cambridge Mass, 1995.

BERMUDEZ, Jose Luiz. Thinking without words. Oxford Press, 2003.

CAMPBELL, John. The role of physical objects in spatial thinking. In: EILAN, N (org). Spatial representation. Oxford Press, 1993.

CLARK, Andy. Supersizing the mind. Oxford Press, 2008.

CHALMERS, David, CLARK, Andy. The extended mind. In: Supersizing the mind. Oxford Press, 2008a 
CRANE, Tim. The Nonconceptual Content of Experience. In: The contents of experience. Cambridge Press, 1992.

CUSSINS, Adrian. Experience, Thought and Activity. In: Essays in nonconceptual content. Cambridge Press, 2003.

DAVIDSON, Donald. Action, Reasons and Causes. In: Essays on action and events. Oxford Press. 1963.

DAVIDSON, Donald. Psychology as Philosophy. In: Essays on action and events. Oxford Press., 1974.

DAVIDSON, Donald. Paradoxes of Irrationality. In: Problems of rationality. Oxford Press. 1982.

DAVIDSON, Donald. Responses to Barry Stroud, John McDowell and Tyler Burge. In:

Philosophy and phenomenological research. Vol. LXVII, n.3, 2003

DAVIDSON, Donald. Aristotle's action. Truth, Language and History. Oxford Press, 2001.

DENNETT, Daniel. Brainstorms. Mit Press, 1978.

DOKIC, Jerôme. L'action située et le principe de Ramsey. In: Raisons pratiques 10: L'Ecole des Hautes Etudes en Sciences Sociales, p. 131-155, 1999.

DUMMETT, Michael. Origins of analytical philosophy. Harvard Press, 1993.

EVANS, Gareth. The varieties of reference. Oxford: Claredon, 1982.

GERGELY, Gyorg; SZABRÓ, Biro. Taking the intentional stance at 12 months of age. Cognition 56. p. 165-193, 1995.

GIBSON, Jerome. The ecological approach to visual perception. Londres: IEA, 1986.

HURLEY, Susan. Consciousness in action. Harvard Press, 1998.

MILNER, David; GOODALE, Melvyn. The visual brain in action. Oxford Press, 2006

MERLEAU-PONTY, Maurice. Fenomenologia da Percepção. Martins Fontes, 1998.

NÖE, Alva. Action in Perception. Mit Press, 2004.

NÖE, Alva. Out of our heads. Hill and Wang, 2009.

PEACOCKE, Christopher. Intuitive mechanics, psychological reality and the idea of a material object. In: EILAN, N (org). Spatial representation. Oxford Press, 1993.

PEACOCKE, Christopher. Does perception have a nonconceptual content?. Journal of Philosophy 98: 239-264, 2001

PERRY, John; ISRAEL, David; TUTYA, Syun. Executions, Motivations and 
Accomplishments. The philosophical review, n. 102, p. 1-34, 1993.

POVINELLI, Daniel. Folk physics for apes. Oxford Press, 2003.

SPELKE, Elizabeth; ROSANDER, Kersting. Predictive action in infancy: tracking and reaching for moving objects. MIT-Press, 1998.

TSAKIRIS, Manos; HAGGARD, Patrick. Neural, Functional, and Phenomenological Signatures of Intentional Actions. In. Naturalizing intention in action. Mit-Press, 2006.

YANTIS, Steven; JONIDES, John. Abrupt visual onsets and selective attention: voluntary versus automatic allocation. Journal of experimental psychology, 1990.

YANTIS, Steven. Visual attention: Bottom up versus Top-Down. Current biology, 2004.

WAKEFIELD, Jerome; DREYFUS, Hubert. Intentionality and the phenomenology of action. In. LEPORE; GULICK (orgs). John Searle and his criticis. Cambridge Mass, 1991. 